\title{
O.S.P.
}

L'orientation scolaire et professionnelle

$30 / 3 \mid 2001$

Juger et être jugé : perspectives psychosociales

\section{C., Delory Monberger. Les histoires de vie : de \\ l'invention de soi au projet de formation.}

Paris : Anthropos.

Thierry Soncarrieu

\section{(2) OpenEdition}

\section{Journals}

Édition électronique

URL : http://journals.openedition.org/osp/5167

DOI : $10.4000 /$ osp. 5167

ISSN : 2104-3795

Éditeur

Institut national d'étude du travail et d'orientation professionnelle (INETOP)

Référence électronique

Thierry Soncarrieu, « C., Delory Monberger. Les histoires de vie : de l'invention de soi au projet de formation. ", L'orientation scolaire et professionnelle [En ligne], 30/3 | 2001, mis en ligne le 20 juillet 2016, consulté le 16 décembre 2020. URL : http://journals.openedition.org/osp/5167 ; DOI : https:// doi.org/10.4000/osp.5167

Ce document a été généré automatiquement le 16 décembre 2020.

() Tous droits réservés 


\title{
C., Delory Monberger. Les histoires de vie: de l'invention de soi au projet de formation.
}

\author{
Paris : Anthropos.
}

Thierry Soncarrieu

\section{RÉFÉRENCE}

Paris : Anthropos.

1 Rémi Hess, Professeur à Paris VIII et Directeur de thèse de Christine Delory Monberger présente cet ouvrage comme fondateur sur les histoires de vie. Le propos peut paraître étonnant dans la mesure où les pratiques concernant les histoires de vie fonctionnent en sciences sociales depuis plus de vingt ans et que ce courant s'inscrit dans une tradition philosophique et littéraire qui remonte à des centaines d'années. Cet ouvrage, paru en avril 2000, est fondateur parce qu'il institue la démarche : il ouvre des pistes, souligne des enjeux nouveaux et ordonne les autres ouvrages, très nombreux sur ce sujet, selon une perspective historique et anthropologique.

2 Dans une première partie intitulée " pratiques et invention de soi ", Christine Delory Monberger propose un itinéraire historique des formes sous lesquelles l'occident a représenté et s'est représenté la dimension individuelle de l'existence humaine de l'antiquité jusqu'au $19^{\prime}$ siècle. Les différentes formes de récit de soi dépendent des conceptions, différentes à travers les siècles, de l'espace public et de l'espace privé, de la relation de l'homme à la société, du rapport de l'homme à lui-même. Aborder les récits de vie conduit à soulever des questions philosophiques de première importance. L'invention de soi, c'est-à-dire l'émergence d'une parole singulière, capable de construire une figure de soi irréductible à tout autre, dépend des environnements socio-historiques et des pratiques de soi. Chaque culture, chaque époque, connaît des modes de relation à soi différents. 
3 D'un point de vue méthodologique, Christine Delory Monberger s'appuie sur la théorie des moments. La vie d'un individu ne se déroule pas de façon linéaire et continue ; il (re)connait des moments constitutifs de son existence (le moment de la famille, de l'amour, du travail...). Dans les récits de vie, on procède à des thématisations : on cristallise, on rassemble, on globalise autour de certains moments. Christine Delory Monberger distingue ainsi sept moments différents des histoires de vie dans la littérature, sept moments significatifs où elle a entendu une voie singulière, décelé une posture nouvelle de la parole de soi.

4 Le premier moment est celui de l'homme sur la place publique : le citoyen. Sur l'agora ou le forum, l'homme grec ou romain n'existe que par sa citoyenneté. Néanmoins, le genre littéraire majeur qu'est l'Éloge laisse une certaine place aux Correspondances (par exemple les lettres d'Atticus de Ciceron) où se manifeste déjà une forme de retour sur soi, un "souci de soi » selon l'expression de M. Foucault. Le 2e moment des histoires de vies, c'est la reconnaissance de l'intériorité : l'homme face à Dieu, avec une première figure emblématique de l'autobiographie : Saint Augustin. A partir de l'analyse de ses Confessions, P. Ricoeur a proposé la distinction toujours moderne de l'identité mêmeté et de l'identité ipséité. La " construction du privé » (3e moment) se développe, durant le Moyen Âge. L'histoire de mes malheurs d'Abélard constitue le pendant profane de l'oeuvre de St Augustin. La Renaissance exhorte l'aventure individuelle (4' moment). Les oeuvres de Rabelais et Montaigne prônent l'autonomie du sujet et sa responsabilité. Avec la Réforme, se développe une certaine individualisation du rapport à Dieu et une certaine conscience de soi. Contre la codification extrême de la Cour de Louis XIV, la défense de l'intime constitue le 5' moment. La notion de " for intérieur " se développe. Sur le plan littéraire, les Mémoires (La Rochefoucauld) et les correspondances intimes (Mme de Sévigné) annoncent le temps des confessions. Après les romans d'apprentissage (ex. Wilhem Meister de Goethe), qui permettent la mise en intrigue des récits de vie, l'empirisme qui affirme le primat de l'expérience sensible et le piétisme qui recommande aux croyants de "s'interroger la plume à la main sur le sens de leur vie » se conjuguent pour faire de l'autobiographie un genre littéraire qui culmine avec les Confessions de J.-J. Rousseau. Enfin, contre la domestication du monde et le secret instaurés par la société bourgeoise du XIX' siècle, " l'écriture du Moi » (7' moment) qui est d'abord le fait des femmes (journaux intimes), envahit tout l'espace littéraire (Chateaubriand, G. Sand, Stendhal...).

5 Il est important d'indiquer la place prépondérante de la littérature dans les modèles d'histoires de vie pour souligner que le récit de vie est d'abord une oeuvre de langage.

Dans une 2' partie, C.D.M. présente les appropriations successives des histoires de vie par les sciences sociales. En effet, à partir du XIX' siècle, l'histoire de vie devient « objet de science ". C.D.M. a vécu en Allemagne et connaît bien la culture germanique. Elle développe l'apport de l'herméneutique et insiste notamment sur les travaux de Wilhem Dilthey, peu connu en France et dont l'influence semble pourtant considérable. Très influencé par le romantisme allemand qui accorde une place fondamentale à l'être intérieur, Dilthey est un des premiers à s'intéresser rigoureusement à la manière dont l'homme transforme sa vie vécue en histoire de vie (en lui donnant une forme et un sens). Ces travaux ont fourni un cadre conceptuel aux histoires de vie et ont rapidement suscité des recherches universitaires en Allemagne. L'autre influence majeure vient des U.S.A. Au début du XX' siècle, l'approche sociologique des histoires de vie commence avec l'école de Chicago : pour comprendre les nouveaux 
comportements citadins, les sociologues vont étudier des groupes étrangers, marginaux, déviants qu'ils considèrent comme " des loupes grossissantes ». Le paysan polonais en Europe et en Amérique : récit d'une vie de migrant de William Thomas et Florian Znaniecki constitue l'ouvrage de référence en la matière. Christine Delory Monberger passe ensuite en revue les nombreux courants théoriques qui sont venus enrichir les histoires de vie : sont ainsi développés, " l'interactionisme symbolique ", " l'ethnométhodologie » (Alfred Schutz) et " l'analyse lexicale des conversations ». Au confluent de ces différentes approches, paraît en 1961 un best-seller sociologique qu'elle analyse également : les enfants de Sanchez d'Oscar Lewis.

7 Le dernier tiers de l'ouvrage est consacré aux perspectives contemporaines. L'auteur présente les axes majeurs de certains courants importants, issus dans la mouvance de 1968, de la phénoménologie, de la théorie de l'action et de la responsabilité qui fait de l'homme « le producteur de sa propre vie».

8 L'enquête ethnosociologique, notamment illustrée par Daniel Bertaux, a pour objectif, à travers le récit d'une vie, de saisir une réalité sociale plus large (ex. la boulangerie...). Dans l'ethnobiographie, en revanche, le récit de vie est considéré comme objet de connaissance en lui-même. (cf. Le cheval d'orgueil de P.-J. Héliaz). Elle présente l'herméneutique objective, plus développée en Allemagne qu'en France, (qui a nourri les travaux de P. Ricœur et, plus récemment de C. Dubar et D. Demazière) et l'analyse narrative-biographique. Elle termine par les histoires de vie en formation qui ont pour but la reconstruction du sujet. Elle indique les présupposés de ces formations :

- l'histoire de vie (racontée) n'est pas la vie mais une « reconfiguration » (Ricoeur), une « synthèse de l'hétérogène ",

- le récit est un « acte adressé » qui se fait pour et avec quelqu'un,

- cette histoire n'est par définition jamais finie, toujours réinventée...

La vraie question est celle de l'identité du narrateur : « tout le problème est de savoir si l'histoire de vie présuppose un sujet cohérent et unifié autour d'une intention ou d'un projet

préalable ou si elle l'aide à le construire ». Elle cite les grands noms de l'espace francophone qui ont tenté de répondre à cette question : Gaston Pineau (Montréal), Pierre Dominicé (Genève), Guy de Villers (Louvain). Elle consacre quelques pages à Vincent de Gaulejac dont la formation : " roman familial et trajectoire sociale ", a pour but de permettre au stagiaire de se comprendre comme le produit d'une histoire dont il cherche à devenir le sujet. Elle présente sa propre démarche, fondée sur les concepts " d'interculturalité " et de " moment biographique ", qu'elle appelle les "ateliers biographiques de projet ». La théoricienne des histoires de vie est aussi praticienne.

Cet ouvrage, fondamental pour les psychologues et les sociologues, spécialistes des récits de vie, soulève des questions et dégage des perspectives historiques, littéraires et philosophiques qui devraient intéresser, au-delà du cercle des experts, un large public ; d'autant que les qualités d'écriture, la clarté du propos et pour tout dire le style de Christine Delory Monberger, accentuent le plaisir de la lecture. 